\title{
Influence of the Temperature as an Environmental Factor on the Electrophysical Behavior of Flexible Polymeric Luminescent Devices
}

\author{
Mariya Aleksandrova \\ Department of Microelectronics, Technical University of Sofia, Kliment Ohridski Boulevard 8, Building 1, Room 1000B, \\ 1000 Sofia, Bulgaria \\ Correspondence should be addressed to Mariya Aleksandrova; m_aleksandrova@tu-sofia.bg
}

Received 30 May 2014; Accepted 28 August 2014; Published 10 September 2014

Academic Editor: Denis Dowling

Copyright (C) 2014 Mariya Aleksandrova. This is an open access article distributed under the Creative Commons Attribution License, which permits unrestricted use, distribution, and reproduction in any medium, provided the original work is properly cited.

\begin{abstract}
The effect of operational temperature on the electrophysical properties of polymer based electroluminescent structures is examined. For this purpose thin film of light-emitting semiconductor polyphenylenevinylene derivative is deposited between two indium-tin oxide (ITO) electrodes. DC current-voltage $(I-V)$ characteristics of the fabricated devices ITO/polyphenylenevinylene derivative PPV-D/ITO are measured at varying ambient temperatures, ranging from room temperature $\left(25^{\circ} \mathrm{C}\right)$ to $70^{\circ} \mathrm{C}$. Several important electrical parameters like a trap factor, traps activation energy distribution, free carriers' density, trapped carriers density, and effective mobility are estimated from measured temperature dependent $I-V$ curves. Such analysis of the charge transport process in polymer devices may give information needed for optimization of the existing structures.
\end{abstract}

\section{Introduction}

A great number of potential applications of organic semiconductor and polymeric materials for use in electronics and optoelectronic devices such as field-effect transistors, solar cells, and organic light-emitting diodes are found [1]. Conjugated polymers are especially modern topic in the research and developing area in this field [2]. Many soluble and semiconducting luminescent polymers with great variety of electrooptical properties were recently synthesized [3]. However, degradation of these devices with respect to temperature and the aging effect are still serious problems. To improve temperature stability and to improve device's performance, it is very important to have a good understanding of the physical phenomena taking place in polymer layers in these devices, such as charge carriers transport, concentration and energy distribution of traps, charge carrier mobility, and other factors with respect to the temperature [4].

Various models are applied to explain different transport mechanisms in organic semiconductors at different modes of the supplied voltage. Among them, two models are used most frequently to explain the $I-V$ characteristics: (a) the trapping model with space-charge-limited current (SCLC) [5] and (b) the field dependent mobility model [6]. The trapping model assumes that there is a certain distribution of traps, called localized states, where the free charge carriers can be trapped. The trapped carriers may be released after some period due to either temperature or other excitations to contribute to the polymer conduction. This model is applied for relatively low voltages, where only temperature dependence of the mobility is considered and electrical field activation is ignored. The field dependent mobility model assumes exponential dependence of mobility on the square root of electric field. There are also several models, concerning determination of defect types (traps), according to their nature (structural defects or impurities) [7]. Among them thermally stimulated luminescence [8] and thermally activated current [9] are more popular.

Poly(9,9-di-(2-ethylhexyl)-9H-fluorene-2,7-vinyle-ne) (PPV-D) is a promising p-type conjugated polymer for luminescent applications [10]. PPV-D has attracted research attention due to its ease of synthesis and relatively good environmental stability. Because it is relatively novel material very limited information is available in scientific literature 

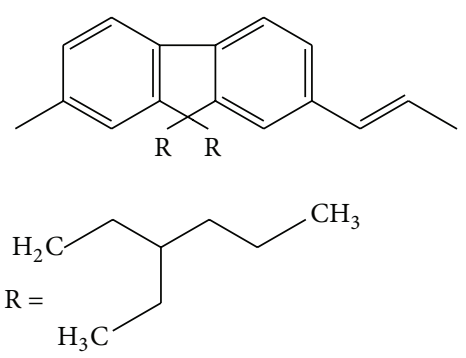

FIGURE 1: Molecular structure of the used light-emitting polymer.

about its electrophysical properties and especially as a function of temperature. The electrical behavior of PPV-D at temperatures higher than room temperature does not seem to have been reported yet.

In this study ITO (indium-tin oxide)/PPV-D/ITO unipolar structures were fabricated and characterized by electrical measurements as a function of the temperature. The observed characteristics were explained on the basis of the already proposed models, assuming that the traps are exponentially distributed in energy band gap of the PPV-D, which was confirmed later by thermally activated current (TAC) measurements. This was made for estimation of the holes traps concentration, ratio of free and trapped charge carriers, trap factor, and their variation at different temperatures.

\section{Materials and Methods}

2.1. Preparation of the Polymeric Structures. In this work a highly pure form of commercially available yellow-orange emitting color PPV-D was used for the fabrication of the films. Figure 1 shows its molecular structure.

The solution with concentration $8 \mathrm{mg} / \mathrm{mL}$ was prepared in chloroform at room temperature $\left(25^{\circ} \mathrm{C}\right)$. The polymer film was deposited on spin coater at angular speed 1100 reverses per minute for spinning time of 40 seconds onto ITO covered polyethylene terephthalate (PET) foil. ITO film with thickness of approximately $148 \mathrm{~nm}$ was grown by vacuum reactive r.f. sputtering at $60 \mathrm{~W}$ sputtering power and $2.5 \cdot 10^{-2}$ Torr total partial pressure (argon + oxygen) in the vacuum chamber. Finally, ITO cathodes were deposited through shadow mask on the polymer at sputtering power of $60 \mathrm{~W}$ to prevent polymer thermal degradation. Formed 8 active areas with dimensions of $2 \mathrm{~mm} \times 3 \mathrm{~mm}$ allow conduction of several measurements on one sample and receiving of statistically true information. On each of both substrate holders in the vacuum chamber 3 substrates can be aligned. In this way 6 samples with 8 active areas or 48 numbers of PPV-D/ITO structures were tested under each experimental condition. Single type of injected charge carriers (holes) is provided for better differentiation of the traps influenced on the holes and those influenced on the electrons. The PPV-D thickness was about $150 \mathrm{~nm}$. Before measuring, copper wires $(\mathrm{Cu})$ were led out from the two electrodes by using high conductive silver (Ag) adhesive paste with no drying. Cross-section view of a finished structure is shown on Figure 2.

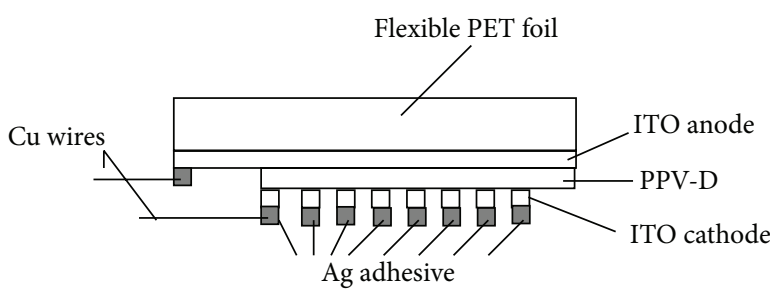

Figure 2: Cross-section view of the flexible ITO/PPV-D/ITO structure.

2.2. Methods for Investigation of the Prepared Organic Structures. The exponential trap distribution model was first proposed by Mark and Helfrich [11]. It was assumed that the free carrier concentration ( $p_{o}$ in our case) is much less than the trapped carrier concentration $p_{t}$ and the trap distribution $h$ as a function of energy $E$ is given as

$$
h(E)=\frac{N_{t}}{E_{c}} \exp \left(-\frac{E_{t}}{E_{c}}\right),
$$

where $N_{t}$ is the density of traps, $E_{t}$ is energy of discrete local level, and $E_{c}$ is the characteristic constant expressed as characteristic temperature $T_{c}, E_{c}=k T_{c}$, where $k$ is the Boltzmann constant. Traps are locations arising from damaged chain bonds in the polymer or impurities; they are called localized states and very often capture free charge carriers, playing a very important role in the conduction and recombination processes in the electroluminescent polymers [12].

Based on the exponentially distributed traps theory, at given voltage $V$ the current $I$ through a polymer layer having thickness $d$, relative permittivity $\varepsilon_{s}$, and density of states in the valence band $N_{v}$ is given by [13]

$$
I=q^{1-l} \cdot \mu_{p} \cdot N_{v} \cdot\left(\frac{2 l+1}{l+1}\right)^{l+1} \cdot\left(\frac{l \varepsilon_{s}}{(l+1) N_{t}}\right)^{l} \cdot \frac{V^{l+1}}{d^{2 l+1}},
$$

where $l=T_{c} / T$ and $T$ is absolute temperature; $q$ is electrical charge. In other words $I-V$ characteristics may follow the dependence $I \sim V^{m}$, where $m=l+1$. Thus, the experimental $I-V$ characteristics may be used to determine the electrical parameters of a conducting polymer by employing (2) to its different regions of operation.

Regarding TAC measurements, the used methodology of measurement of thermostimulation of charge carriers follows the following steps: (1) cooling of the sample to lower than room temperature, but higher than zero degrees Celsius to avoid condensation; (2) applying of forward (turn-on) voltage to cause current flow for filling the traps; (3) cyclic heating to a certain temperature and current measurement at this temperature interval; (4) repeating of the whole cycle but at higher heating temperature until maximal temperature is achieved (which is the maximum allowable for the polymer). The measured current is plotted as a function of the applied temperature change. For association of the energy position of the traps with the obtained signal its initial slope at every measurement step must be taken into account. This method was chosen, because in unipolar structures there is no luminescence and TSL method is inapplicable. 


\section{Results and Discussion}

The prepared structures were investigated by measurement of $I-V$ characteristics at different ambient temperatures. For this purpose the samples were put in thermostat chamber. The temperature regulation circuit consists of contact thermometer (CT) and circuit closer (K1), which switch over the heater in the chamber. The range of the controlled temperatures is from room temperature $\left(25^{\circ} \mathrm{C}\right)$ only to $70^{\circ} \mathrm{C}$, because the polymer's and plastic substrate's degradation temperature is approximately $80-85^{\circ} \mathrm{C}$. The precision of the temperature maintenance was $\pm 1.5^{\circ} \mathrm{C}$. The prepared ITO/PPV-D/ITO structures were attached to the apparatuses with Teflon wires, holding out against high temperatures and not allowing inducting of disturbances.

The block diagram of the test circuit is shown in Figure 3. For measurement of the $I-V$ characteristics regulating voltage source was used with working range from $0 \mathrm{~V}$ to $12 \mathrm{~V}$. The adjustment of the voltage applied to the device electrodes was realized by voltage attenuator which consists of reference voltage source $E_{G}$, multiturn trimmer-potentiometer $R_{p}$, and voltage follower with precise operational amplifier OP177 (from Analog Devices). The voltage and current measurement were realized, respectively, with digital voltmeter VC820 (from Voltcraft) with accuracy 4.5 digits and precise picoampermeter Keithley 6485.

The obtained $I-V$ characteristics as a function of temperature for ITO/PPV-D/ITO devices are shown in Figure 4. They considered a forward bias operation, since the reverse voltage behavior is standard junction behavior with leakage current, increasing with the temperature. It is not expected to be higher than $\mathrm{nA}$ values, even at $70^{\circ} \mathrm{C}$, because the conductivity here is lower in comparison with an inorganic semiconductor. It is evident from Figure 4 that the electrical response strongly varies with temperature. In the low voltage Region 1 under $80 \mathrm{mV}$ (R1), a negligible amount of current is flowing. Zoom view of R1 (not displayed) shows that the characteristics are almost linear. At higher voltages (above $\sim 100 \mathrm{mV}$ ) the current follows $I \sim V^{2}$ (square law in Region 2 (R2)), indicating presence of space-charge-limited conduction [14].

Lampert's theory of SCLC predicts that valuable information could be obtained from $I-V$ characteristics of organic semiconductors while operated in R2 [15]. For this region (2) is transformed to

$$
I=\frac{9}{8} \varepsilon_{s} \theta \mu_{p} \frac{V^{2}}{d^{3}},
$$

where $\theta$ represents the trap factor, which is defined as the ratio of free hole/electron concentration $p_{o} / n_{o}$ to the total carrier concentration $\left(p_{o}+p_{t}\right)$ or $\left(n_{o}+n_{t}\right)$ and for a $p$-type polymer film is

$$
\theta=\frac{p_{o}}{p_{o}+p_{t}}
$$

where $p_{t}$ is concentration of trapped holes. At $25^{\circ} \mathrm{C}$ and $50^{\circ} \mathrm{C}$ the $I \sim V^{2}$ dependence can be seen clearer, because the electric field is predominant component of excitation over the heat energy for causing current flow. The threshold voltages in these cases are in the middle of $\mathrm{R} 2$ zone (resp., $1.5 \mathrm{~V}$ and $0.2 \mathrm{~V}$ ). With temperature increasing to $60^{\circ} \mathrm{C}$ and $70^{\circ} \mathrm{C}$, the heat energy becomes predominant for activation of the conductivity process, so the voltage is almost negligible (near to $0.1 \mathrm{~V}$ ) and it is needed only for causing charge carriers movement from electrode to electrode. $I \sim V^{2}$ dependence is still valid but the curve before the transition point is shorter in comparison with the lower temperatures $\left(25^{\circ} \mathrm{C}\right.$ and $50^{\circ} \mathrm{C}$ ). Experimentally, the value of trap factor $\theta$ could be calculated from the ratio of currents $I 1$ and $I 2$ at the beginning and the end of square law regions, respectively, for each characteristic. For example, at temperature $25^{\circ} \mathrm{C}$ the current value $I 1$ at the beginning of $\mathrm{R} 2$ range is $0.8 .10^{-8} \mathrm{~A}$ and it increases to $0.8 .10^{-4} \mathrm{~A}$ at the end of the $I-V^{2}$ range, so the trap factor is $0.1 \cdot 10^{-3}$. The results for $\theta$ obtained at different temperatures are summarized and illustrated in Figure 5. The initial value of the trap factor increases gradually with the temperature and after that there is a steep increase (occurring at about $60^{\circ} \mathrm{C}$ ). This may be attributed to the fact that at higher temperatures more trapped charge carriers are released from their localized states and play a role in the conduction process as free carriers in the PPV-D film. This means that there exist many traps with similar activation energy, corresponding to $62^{\circ} \mathrm{C}$. This value is calculated to be $128 \mu \mathrm{eV}$ from the dependence between trap activation energies and the temperature.

The energy distribution is given in Figure 6, where the initial part of the curve is well fitted with exponential growing function as was assumed in the beginning. Energy distribution of the traps is spread in relatively wide range of energies from 10 to $130 \mu \mathrm{eV}$. There are no formed single discrete levels. This broad distribution is evidence for structural disorder in the polymer chains and layer imperfections descending from it.

Using (3) and assuming $\theta=1$, the variation in the main charges mobility $\mu_{p}$ as a function of temperature was evaluated from the observed characteristics and plotted in Figure 7. A dielectric constant $\varepsilon_{s}=3$ was used for PPV-D in the calculations [16]. The voltage was chosen in the SCLC region. A value of $4 \mathrm{~V}$ was used, as all characteristics at the different temperatures are in SCLC regime for this voltage.

Figure 7 also shows the variation of effective mobility $\mu_{\text {eff }}$ obtained by incorporation of the trap factor. The effective mobility, which includes the trap factor, is less than mobility of a pristine (trap free) organic semiconductor, because traps induce distortion of the electrical field inside the polymer film and interfere with the movement of the free holes. The effective mobility can be expressed as in [13]. Consider

$$
\mu_{\mathrm{eff}}=\mu_{p} \theta=\mu_{p}\left[\frac{p_{o}}{p_{o}+p_{t}}\right] .
$$

Figure 8 shows the variation of $p_{o}$ and $p_{t}$ as a function of the temperature. These variables were calculated using the transition voltage $V_{\text {tr }}$ [17] between linear region $\mathrm{R} 1$ and square law region R2 (represented in Figure 4) and the trap factor. As can be seen the concentration of free carriers increases considerably at higher temperatures, resulting in a corresponding decrease of trapped carrier concentration in 


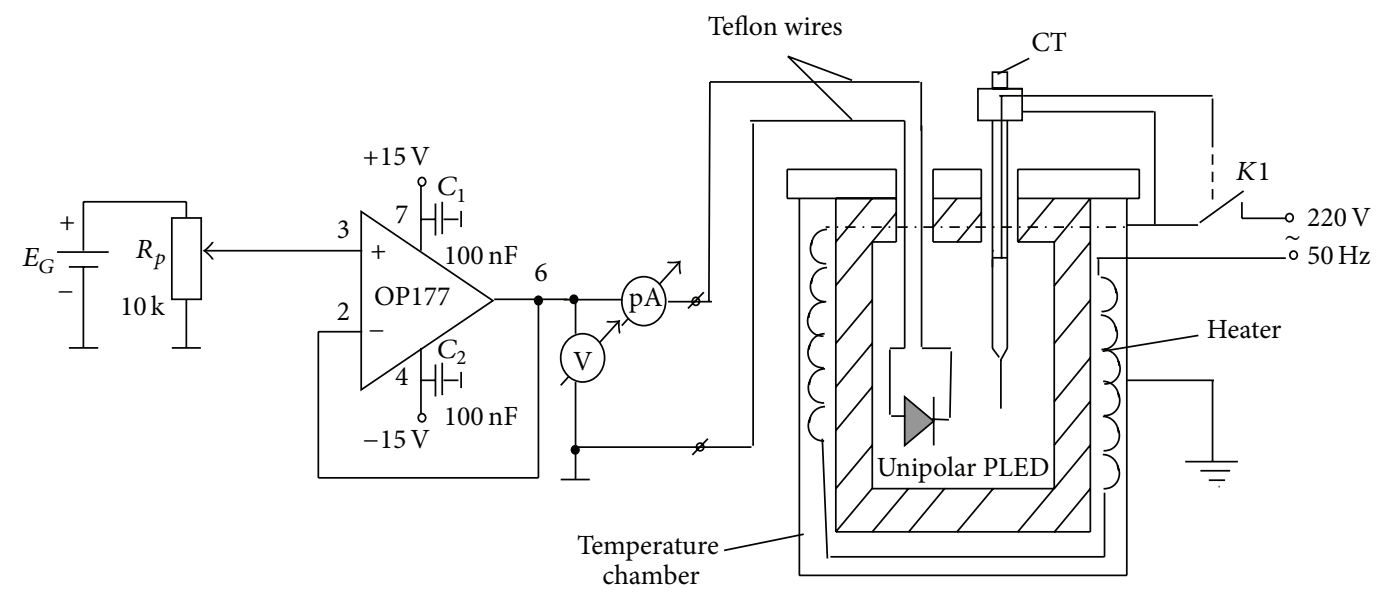

FIGURE 3: Block diagram of the test circuit for $I-V$ characteristics measurement as a function of the temperature.

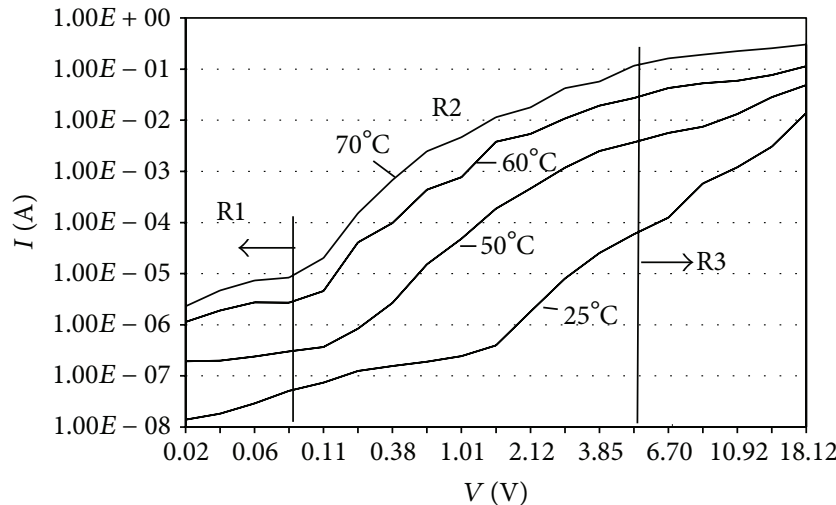

FIGURE 4: $I-V$ characteristics of ITO/PPV-D/ITO organic electroluminescent structure at different temperatures from $25^{\circ} \mathrm{C}$ to $70^{\circ} \mathrm{C}$.

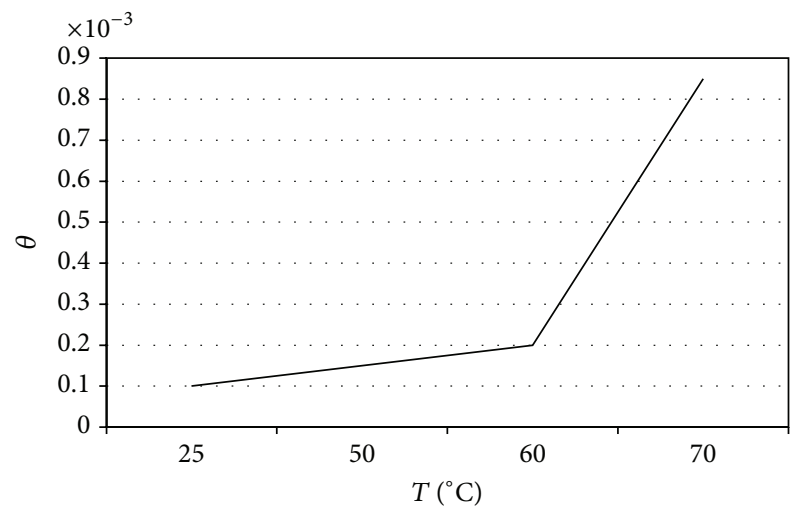

FIGURE 5: Variation of the trap factor with the temperature for ITO/PPV-D/ITO device.

the localized states. The traps which could be present due to impurity or structural defects restrict the charges inside the energy band gap and hence affect the overall hopping process. When temperature increases, it is assumed that the charge is exited and may jump from a localized to an extended state. This could explain the observed higher current at high temperatures in PPV-D based structure depicted in Figure 4.

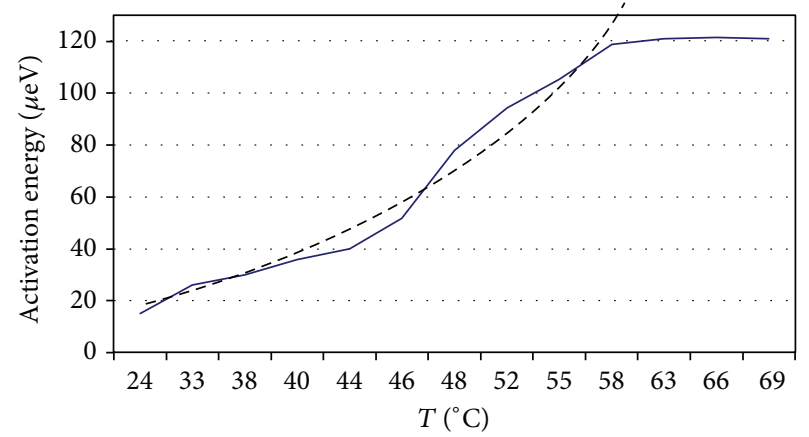

FIgURE 6: Activation energy of traps in polymer based structure consists of spin-coated PPV-D at $1100 \mathrm{rpm}$.

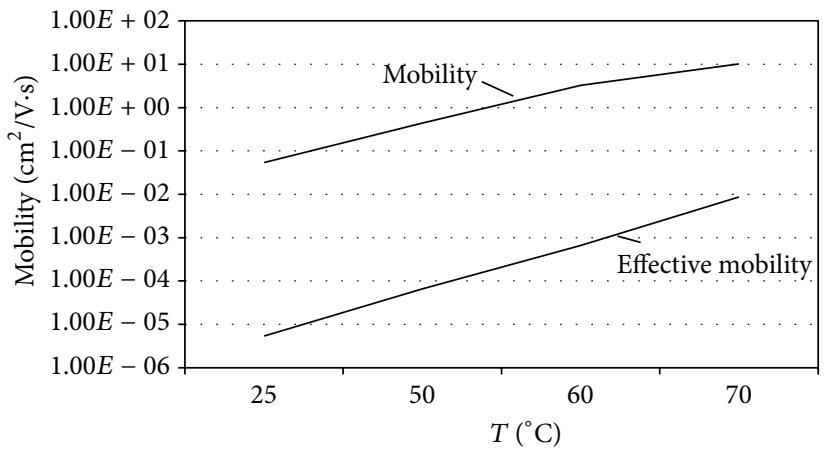

FIGURE 7: Variation of effective mobility and trap free mobility at different temperatures for PPV-D film.

As the trap factor is a ratio between $p_{o}$ and $\left(p_{o}+p_{t}\right)$ it therefore also increases with the temperature as shown in Figure 5. Increasing of the mobility by increasing the temperature may be associated with the energy of the carriers, which is now high enough to allow them to move without falling into a trap.

\section{Conclusions}

The presence of defects in polymer based structures modifies their electrical properties. The identification of 


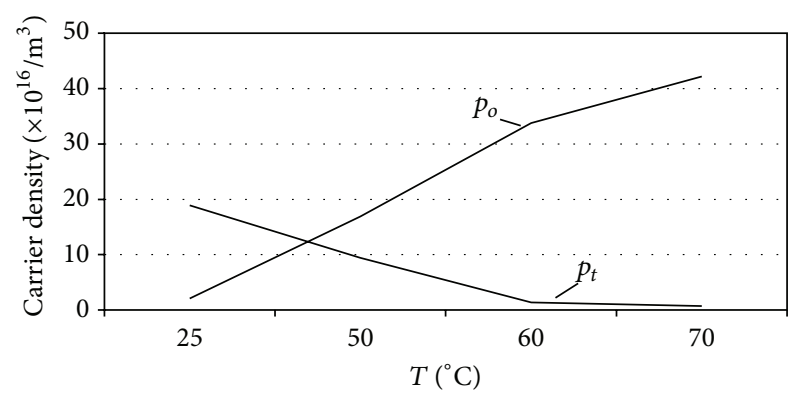

FIGURE 8: Variation of free carrier density $p_{o}$ and trapped carrier density $p_{t}$ for PPV-D film as a function of the temperature.

the mechanisms that dominate charge transport processes may provide the necessary background for the development of new device structures or improvement of the existing ones. A practical approach was applied here for analysis of experimental results obtained by DC conductivity measurements which makes the determination of parameters possible, characterizing the charge transport in polymers used for light-emitting applications. The carrier detrapping from the traps is stimulated by thermal energy at operational temperatures in the range $25-70^{\circ} \mathrm{C}$. It was observed that these devices are nonlinear and the measured characteristics were explained assuming a hopping of free carriers between the energy states presented in the organic film. By applying the trapped space-charge-limited current model on the received $I-V$ curves, the conduction mechanism and different electrical parameters such as trap factor, effective mobility, free carrier density, and trapped carrier density were determined and their temperature dependence was investigated.

\section{Conflict of Interests}

The author declares that there is no conflict of interests regarding the publication of this paper.

\section{Acknowledgment}

This work was financially supported by Project 141ПР000503.

\section{References}

[1] S. Moss and Z. Bao, "Organic optoelectronic materials, processing and devices," in Proceedings of the MRS Symposium Proceeding, Boston, Mass, USA, November 2001.

[2] P. Gaşiorski, E. Gondek, M. Pokladko-Kowar et al., "Methoxyphenyl indenopyrazoloquinoline derivatives as green fluorescent emitters for polymer based electroluminescent devices," Optical Materials, vol. 34, no. 1, pp. 317-321, 2011.

[3] M. Chemli, A. H. Saï, N. Jaballah, J.-L. Fave, and M. Majdoub, "Synthesis and characterization of new electroluminescent poly(p-phenylene) derivative," Synthetic Metals, vol. 161, no. 1516, pp. 1463-1468, 2011.
[4] I. Campbell and D. Smith, "Electrical transport in organic semiconductors," International Journal of High Speed Electronics and Systems, vol. 11, pp. 223-249, 2001.

[5] A. K. Kapoor, S. C. Jain, J. Poortmans, V. Kumar, and R. Mertens, "Temperature dependence of carrier transport in conducting polymers: similarity to amorphous inorganic semiconductors," Journal of Applied Physics, vol. 92, no. 7, pp. 3835-3838, 2002.

[6] S. V. Rakhmanova and E. M. Conwell, "Electric-field dependence of mobility in conjugated polymer films," Applied Physics Letters, vol. 76, no. 25, pp. 3822-3824, 2000.

[7] T. Provder and C. D. Craver, Eds., Polymer Characterization: Physical Property, Spectroscopy, and Chromotographic Methods, vol. 227 of Advances in Chemistry Series, 1990.

[8] A. Kadashchuk, A. Andreev, H. Sitter, and N. S. Sariciftci, "Photo- and thermally stimulated luminescence in highly ordered films of para-sexiphenyl grown by Hot-Wall Epitaxy," Synthetic Metals, vol. 139, no. 3, pp. 937-940, 2003.

[9] M. Aleksandrova, N. Nikolov, and I. Pandiev, "Thermostimulation of charges by peltier element for trap analysis in polymer layers," International Journal of Polymer Analysis and Characterization, vol. 16, no. 4, pp. 221-227, 2011.

[10] Sigma Aldrich, 2012, http://www.sigmaaldrich.com/catalog/ product/aldrich/656267?lang=en\&region=BG.

[11] P. Mark and W. Helfrich, "Space-charge-limited currents in organic crystals," Journal of Applied Physics, vol. 33, no. 1, pp. 205-215, 1962.

[12] R. Schmechel and H. Von Seggern, "Electronic traps in organic transport layers," Physica Status Solidi A, vol. 201, no. 6, pp. 12151235, 2004.

[13] K. P. Nazeer, S. A. Jacob, M. Thamilselvan, D. Mangalaraj, S. K. Narayandass, and J. Yi, "Space-charge limited conduction in polyaniline films," Polymer International, vol. 53, no. 7, pp. 898902, 2004.

[14] S. Nešpůrek, O. Zmeškal, and J. Sworakowski, "Space-chargelimited currents in organic films: some open problems," Thin Solid Films, vol. 516, no. 24, pp. 8949-8962, 2008.

[15] M. A. Lampert, Current Injection in Solids, Academic Press, New York, NY, USA, 1970.

[16] Y.-C. Nah, S.-S. Kim, J.-H. Park, H.-J. Park, J. Jo, and D.-Y. Kim, "Enhanced electrochromic absorption in Ag nanoparticle embedded conjugated polymer composite films," Electrochemistry Communications, vol. 9, no. 7, pp. 1542-1546, 2007.

[17] K. Kao and W. Hwang, Electrical Transport in Solids, Pergamon Press, Oxford, UK, 1981. 

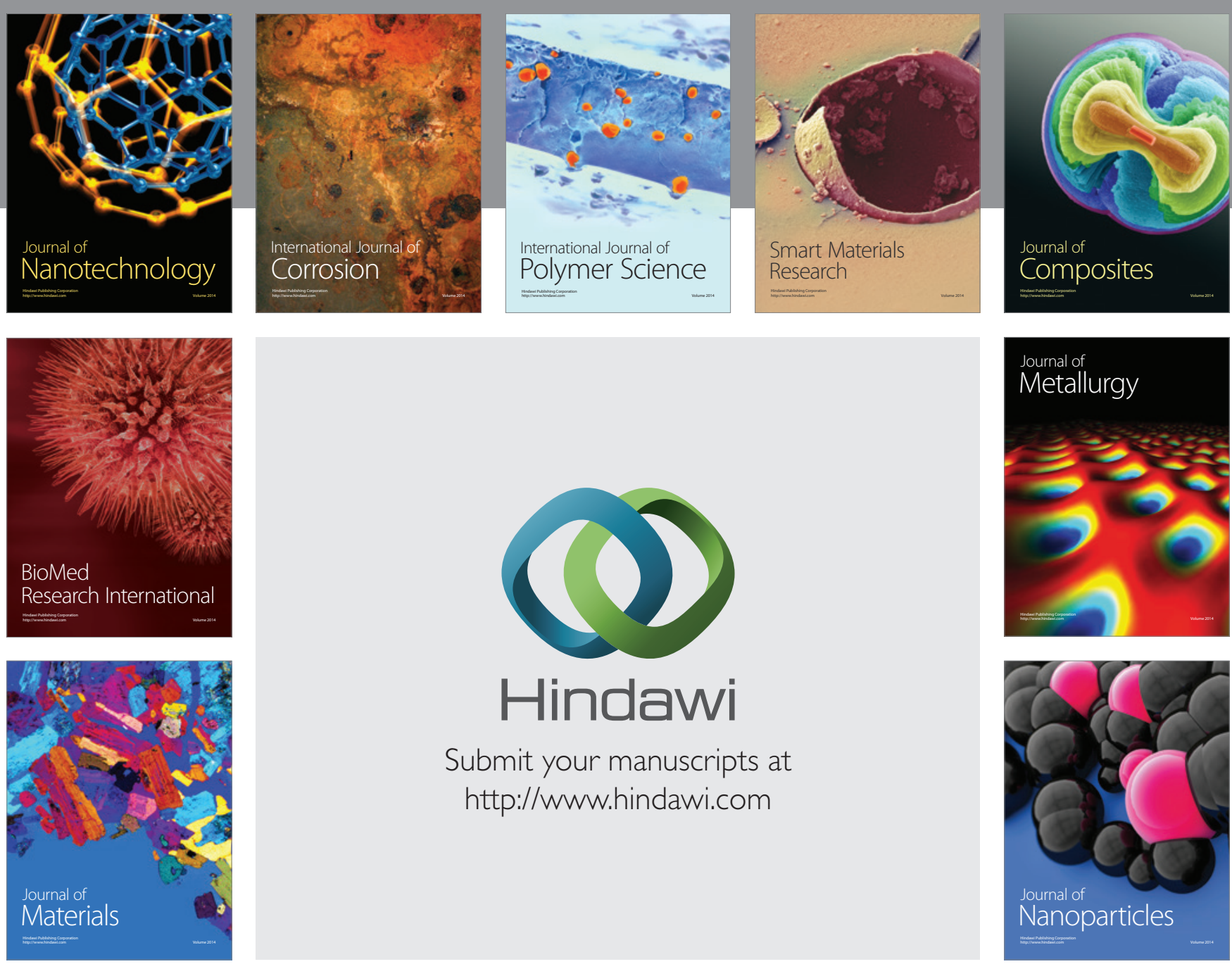

Submit your manuscripts at http://www.hindawi.com
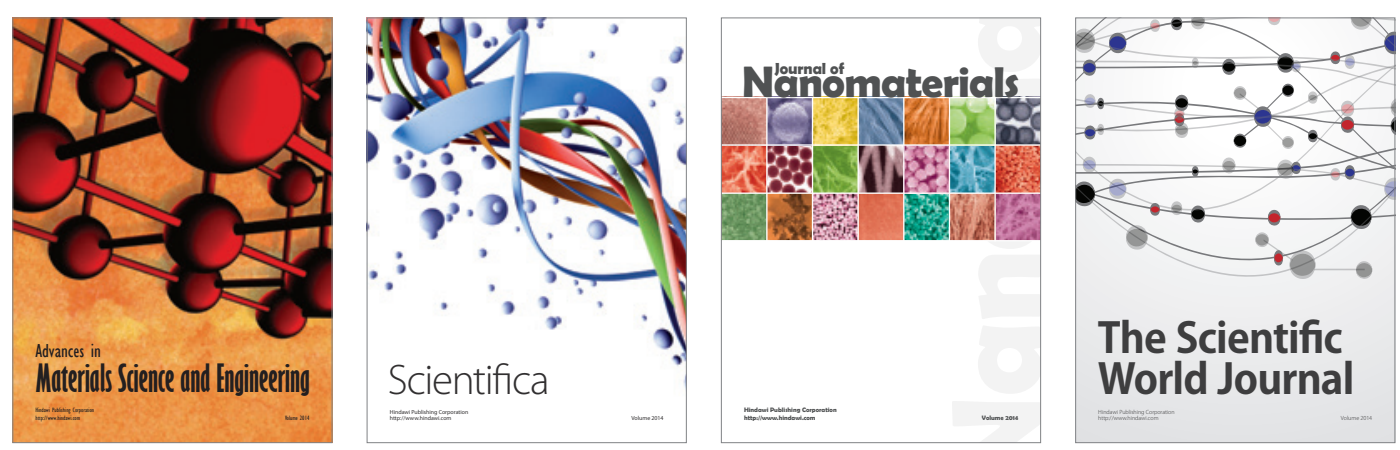

\section{The Scientific World Journal}
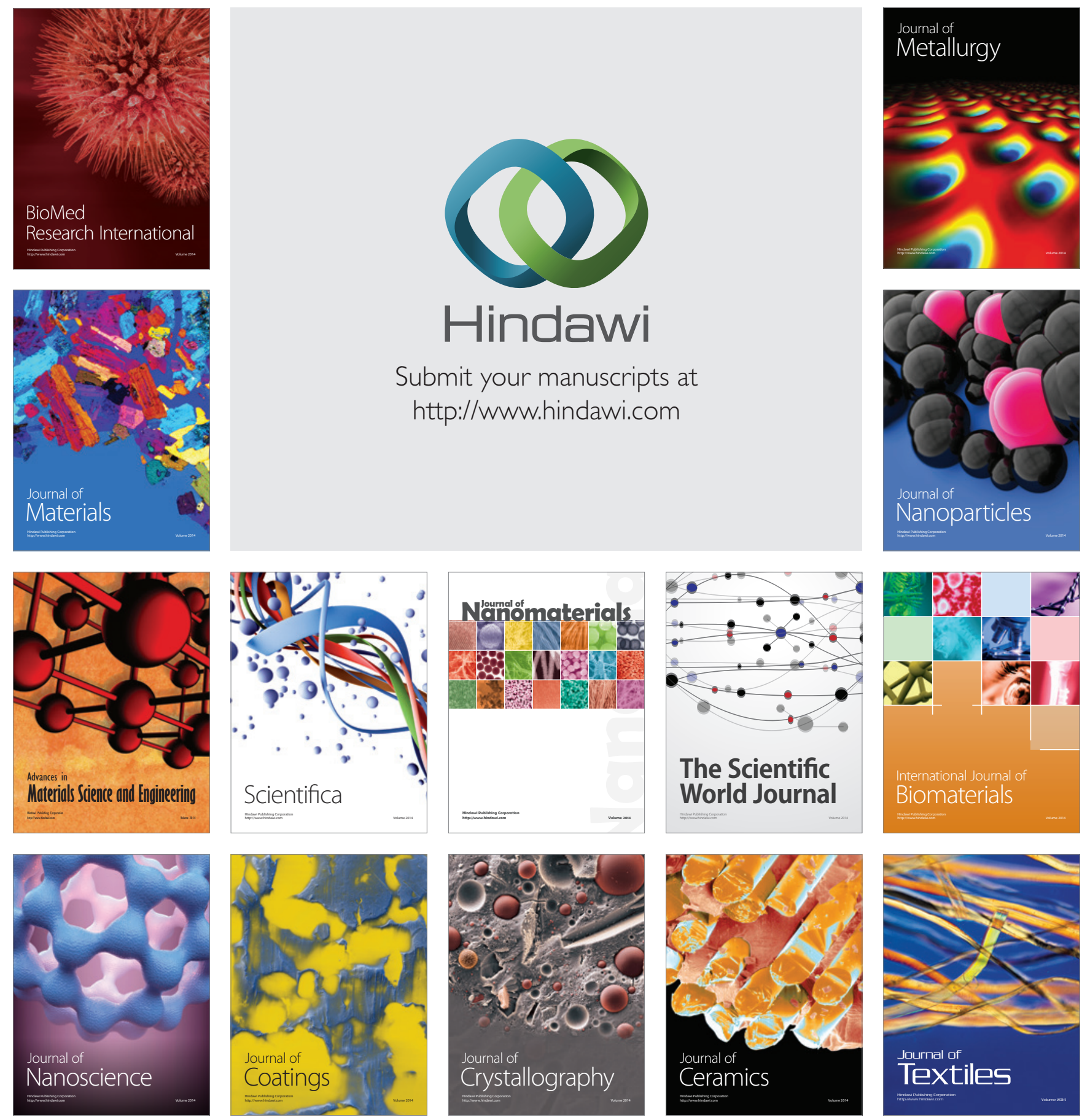